\title{
2. SYNOPSIS
}

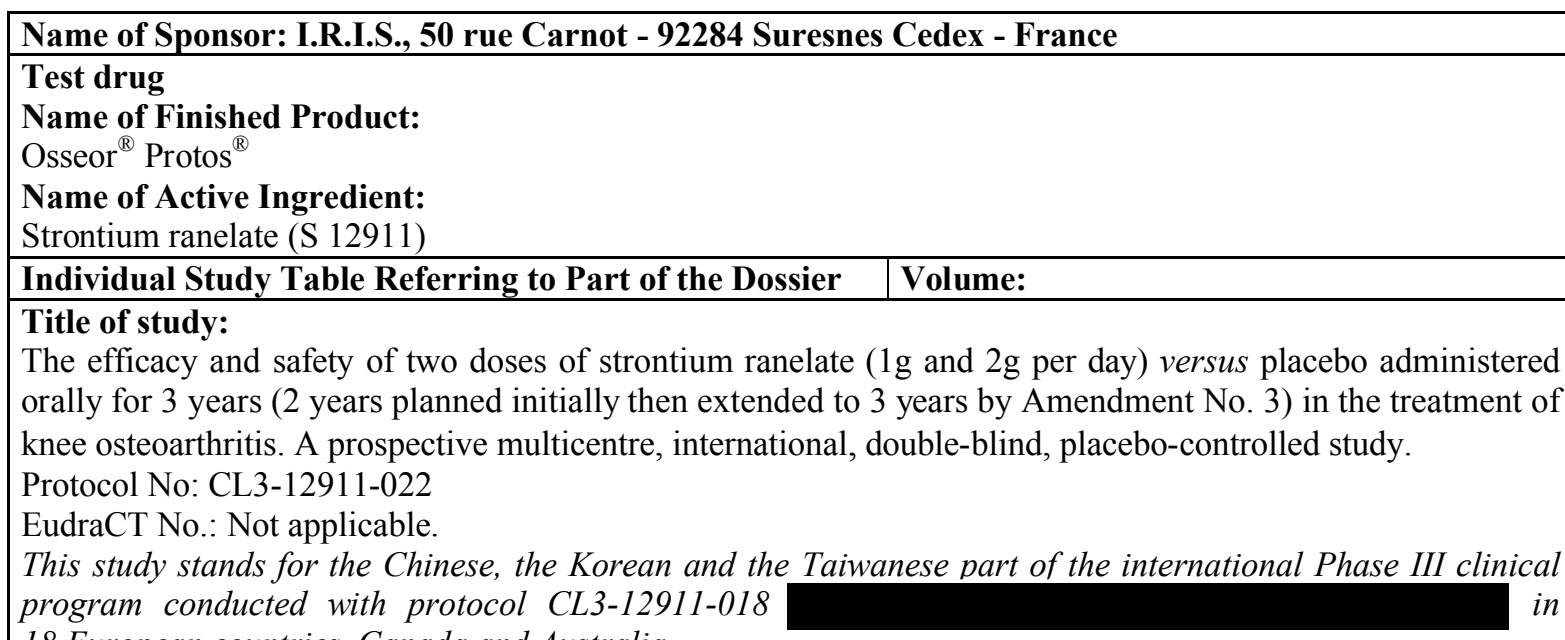
18 European countries, Canada and Australia.

The description of the study protocol given hereafter includes the modifications of the 6 substantial amendments to the protocol.

\section{International coordinator}

\section{Study centres:}

International, multicentre study: 24 centres located in 3 countries included 452 patients: 11 centres in China (328 patients included), 8 centres in Korea (90 patients included), and 5 centres in Taiwan (34 patients included).

\section{Publication (reference): Not Applicable.}

\section{Studied period:}

Initiation date: 27 June 2008 (date of first visit first patient)

Completion date: 05 September 2013 (date of last visit last patient)

\section{Objectives:}

The main objective was to demonstrate the superiority of strontium ranelate ( $1 \mathrm{~g}$ and $2 \mathrm{~g}$ per day) versus placebo against articular cartilage damage progression over two years extended to three years (according to amendment No. 3) in men and women with knee osteoarthritis by measuring changes in cartilage joint assessed by X-ray imaging over the study period.

The secondary objectives were:

To demonstrate the efficacy against pain and functional aspects of the osteoarthritis of the knee.

To assess the efficacy against articular cartilage progression by measuring changes in cartilage volume assessed by Magnetic MRI imaging (in a subset of patients in China only).

To assess the presence and evolution of concomitant hip osteoarthritis.

To assess the presence and evolution of concomitant hand osteoarthritis.

To assess the safety of two different doses of strontium ranelate administered orally for a duration of 24 months amended to 36 months in men and women with knee osteoarthritis.

To describe the pharmacokinetics of two different doses of strontium ranelate in men and women with knee osteoarthritis (in a subset of patients in China only).

To perform a pharmaco-economic evaluation.

To measure the strontium content in bone and cartilage tissues from waste tissue potentially obtained during surgery of the target joint where available, and if possible (according to local regulations), to perform experimental in vitro measurements on the bone and cartilage cells obtained from these waste tissues. In the case where an aspiration of the knee is required during the study, the intra-articular fluid obtained should, if possible, be aliquoted and frozen in order to possibly measure certain cartilage and inflammatory markers, as well as the strontium level in this fluid. 


\section{Methodology:}

International, multicentre, randomised using an Interactive Voice Response System (IVRS), double-blind, placebo-controlled study, with 3 parallel groups (SrRan 1g, SrRan 2g and placebo), with therapeutic benefit, over a 2-year period amended to a 3-year period (amendment No. 3). Study treatments were allocated using a randomisation stratified on centre and gender.

3 Committees were set-up:

- The Executive Committee was in charge of the scientific and ethical aspects of the study and proposed amendments to the study protocol. This committee validated the final results and conclusions of the study.

- The Steering Committee was in charge of practical and technical aspects of the study.

- The Safety Committee composed of experts in the field of medical surveillance, was to inform and/or formulate recommendations in case of unexpected safety issues.

This study was performed in strict accordance with Good Clinical Practice including the archiving of essential documents.

An abbreviated report was written, as the osteoarthritis indication was not finally submitted in Asia.

\section{Number of patients:}

Planned: 315 patients included (210 in China and 105 patients in Korea), ie. for each treatment group: 70 patients in China, 35 patients in Korea, amended to 360 patients (210 included in China, 105 patients in Korea, and 45 patients in Taiwan), i.e. for each group: 70 patients in China, 35 patients in Korea and 15 patients in Taiwan then amended to 450 patients (300 included in China, 105 patients in Korea, and 45 patients in Taiwan), i.e. for each group: 100 patients in China, 35 patients in Korea and 15 patients in Taiwan.

Included: 452 patients (151 in the SrRan $1 \mathrm{~g}$ group, 151 in the SrRan $2 \mathrm{~g}$ group and 150 in the placebo group). Diagnosis and main criteria for inclusion:

Asian, ambulatory, men and women aged $\geq 50$ years, with a primary knee osteoarthritis (OA) of the medial femoro-tibial compartment diagnosed according to the clinical and radiological criteria of the American College of Rheumatology, a presence of Kellgren and Lawrence grading stages II or III on knee X-rays and a Joint Space Width (JSW) between 2.5 and $5 \mathrm{~mm}$ (inclusive limits). A target knee was defined taking into account the Kellgren and Lawrence score, the anatomic compartment, and the Visual Analogue Scale (VAS) value at inclusion, and was then to be followed throughout the study.

\section{Test drug:}

Strontium ranelate given orally as sachet of granules of $1 \mathrm{~g}$ or $2 \mathrm{~g}$ taken as a suspension once daily at bedtime. Batch Nos. Strontium ranelate 1g: L0014482, L0025233, L0028215, L0033069, L0040376; Strontium ranelate 2g: L0014525, L0025235, L0027975, L0033071, L0040378.

Comparator (Reference product and/or placebo):

Matching placebo sachet given orally as a suspension once daily at bedtime.

\section{Duration of treatment:}

From selection visit (ASSE) to inclusion visit (M0): 2-week period without study treatment.

Active treatment period (M0-M36): 24 months extended to 36 months (amendment No. 3) with two additional visits M30 and M36 and one additional phone call at M27.

\section{Criteria for evaluation:}

\section{Efficacy measurements}

\section{Primary efficacy endpoint:}

Radiographic progression of knee osteoarthritis by assessment of the JSW at selection, M12, M24 and M36 visits (standardised assessment and centralised reading by PMO, Prevention des Maladies Osseuses, Lyon, France). The measurement of the mean change versus placebo in the minimal JSW of the medial femoro-tibial compartment was determined by X-ray. 


\section{Criteria for evaluation (Cont'd):}

\section{Secondary efficacy endpoints:}

- Radiological and radio-clinical progression of the knee osteoarthritis: number of failures, assessed by knee JSW progression (i.e. JSW loss $\geq 0.5 \mathrm{~mm}$ ) and radio-clinical failure assessed by an index combining knee JSW loss $\geq 0.5 \mathrm{~mm}$ and the WOMAC pain sub-score improvement $(\leq 20 \%)$ at the last post baseline visit.

- Clinical assessments:

- Algo-functional assessment of the target knee by the WOMAC index score (Western Ontario and McMaster Universities Osteoarthritis Index) every 6 months from M0 to M36.

- VAS for the target knee pain scale assessment at selection, M0, and every 6 months up to M36 with a recall period of 48 hours.

- Target knee physical assessment at each visit.

- Time to indication to surgery for target knee joint replacement.

- E-diary filled in by the patient: knee pain flare frequency and intensity, and pain medication consumption through a once weekly phone call.

- Knee Magnetic Resonance Imaging (MRI), assessing the cartilage volume and other parameters (performed in a subset of patients in China only) at M0, M12, M24 and M36 visits.

- Biochemical bone markers: serum Bone ALkaline Phosphatase (bALP) and C-terminal Telopeptides of type I collagen (CTX I) and cartilage markers: serum C Propeptide of type II procollagen (CPII) and urinary C-terminal Telopeptides of type II collagen (CTX II) and one marker of synovial metabolism Hyaluronic Acid (HA), at M0, M3, M6, M12, M24, M30, and M36.

- Hand radiological and clinical assessment:

- Radiologic assessment of hand osteoarthritis at M0 and M36.

- Clinical assessment of hand osteoarthritis.

- Clinical assessement by the investigator of pain in finger joints at M0, M12, M24, and M36 visits and of finger nodes at M0 and M36 visits only.

- Pharmaco-economic assessment (need for: physiotherapy, nursing care sessions, paramedical sessions, additional medical imaging procedures or physician visits, hospitalisation for knee inflammation, total knee replacement, knee arthroscopy, rehabilitation unit admission and water cures, drugs consumption) assessed for a 6-month period prior to inclusion, and at M3 M6, M12, M18, M24, M30 and M36.

Safety measurements:

- Adverse events reported at each visit.

- Vital signs (weight, height, systolic and diastolic blood pressure, heart rate) at each visit.

- Laboratory safety parameters: biochemistry parameters [total alkaline phosphatase, ASAT, ALAT, GGT, blood and urinary creatinine, phosphorus, blood and urinary calcium, sodium, potassium, chloride, albumin, C-reactive protein, CPK (and isoenzymes if CPK above the upper limit of the reference range)] and haematology parameters (blood cell count, haemoglobin, haematocrit, MCV) at selection, M3, M6, M12, M18, M24, M30 and M36 except for sodium, potassium, chloride assessed only at selection, M12, M24 and M36 visits.

- Haemostasis parameters (prothrombin time, activated partial thromboplastine time) assessed in the Chinese population only except for patients under anticoagulant therapy at M0, , M12, M24, and M36.

Pharmacokinetic measurements

To assess the pharmacokinetics (PK) of strontium in patients with knee osteoarthrisis, serum samples were collected in all patients in the morning of M0, M3, M6 and every 6 months up to M36 (M30 and M36 added by amendment). Additionally, at M6 and M18, and added by amendment M30 in a Chinese population subset, the patients were asked to take their daily treatment in the morning during the visit, after the first blood collection. In these patients, an additional blood sample was collected at least $1 \mathrm{~h} 30$ after the morning study treatment intake. At M30, in the same subgroup, an additional blood sample was collected at least $1 \mathrm{~h} 30$ after the first sampling in the morning but without taking any medication before.

Serum concentrations of strontium were assessed by high frequency inductively coupled plasma atomic emission spectrometry (ICP-AES). 


\section{Statistical methods:}

Study outcome: Descriptive statistics were provided.

Efficacy analyses were primarily performed in the Full Analysis Set (FAS) defined as all randomised patients who took at least one dose of the study treatment and who had one assessable baseline and at least one assessable post-baseline evaluation for the JSW [according to the the central reader (PMO - Lyon)].

\section{Primary criterion}

Main Analysis

The JSW (from PMO reading) was expressed as the change from baseline to last post-baseline value. Each SrRan group was compared to the placebo group in the FAS using a general linear with baseline JSW, centre and gender as covariates. Estimates $(\mathrm{E})$ of the difference between adjusted group means and Standard Error (SE) of the estimate were provided with its $95 \% \mathrm{CI}$ and the associated p-value.

\section{Secondary criteria}

Decriptive statistics were provided for secondary criteria:

- Radiological and radio-clinical progression of the knee osteoarthritis.

- Clinical assessments : WOMAC scores for the target knee,VAS knee pain, Knee physical assessment, indication to knee surgery for target knee joint replacement, E-diary (feeling of pain, intensity of pain, the mean numbers of days with pain per week and the pain medication consumption).

- Knee MRI parameters.

- Biochemical cartilage and bone markers.

- Hand radiological and clinical assessment: Hand osteoarthritis X-ray evaluation, algo-functional assessment of hand.

- Hip clinical assessment: functional assessment, VAS pain, and occurrence of hip prosthesis implantation.

- Pharmaco-economic parameters.

\section{Safety analysis:}

Adverse events, laboratory parameters and vital signs were analysed through descriptive statistics.

\section{Pharmacokinetic analysis}

Descriptive statitistics on strontium concentrations were calculated at each sampling time per group.

In a second approach, serum strontium concentrations were analysed using a compartmental population approach, according to a separate protocol. An analysis of the relationship between pharmacodynamic (PD) data (osteoarthritis biomarkers) and strontium exposures were planned to be performed by using a graphical approach. However, these analyses were not finnaly performed. 


\begin{tabular}{|c|c|c|c|c|c|c|c|c|}
\hline \multicolumn{9}{|l|}{ SUMMARY - CONCLUSIONS } \\
\hline \multicolumn{9}{|c|}{ DISPOSITION OF PATIENTS AND ANALYSIS SETS } \\
\hline \multicolumn{9}{|c|}{ Disposition of patients } \\
\hline \multirow{2}{*}{ Status } & \multicolumn{2}{|c|}{ SrRan 1g } & \multicolumn{2}{|c|}{ SrRan 2g } & \multicolumn{2}{|c|}{ Placebo } & \multicolumn{2}{|c|}{ All } \\
\hline & $\mathrm{n}$ & $\%$ & $\mathrm{n}$ & $\%$ & $\mathrm{n}$ & $\%$ & $\mathrm{n}$ & $\%$ \\
\hline Included (randomised) & 151 & 100 & 151 & 100 & 150 & 100 & 452 & 100 \\
\hline In compliance with the protocol & 133 & 88.1 & 130 & 86.1 & 128 & 85.3 & 391 & 86.5 \\
\hline With a protocol deviation at inclusion & 18 & 11.9 & 21 & 13.9 & 22 & 14.7 & 61 & 13.5 \\
\hline Withdrawn from the study due to & 59 & 39.1 & 64 & 42.4 & $\mathbf{5 0}$ & 33.3 & 173 & 38.3 \\
\hline Non-medical reason & 38 & 25.2 & 40 & 26.5 & 33 & 22.0 & 111 & 24.6 \\
\hline Adverse event & 17 & 11.3 & 21 & 13.9 & 14 & 9.3 & 52 & 11.5 \\
\hline Lost to follow-up & 2 & 1.3 & 1 & 0.7 & 2 & 1.3 & 5 & 1.1 \\
\hline Protocol deviation & 2 & 1.3 & 2 & 1.3 & - & - & 4 & 0.9 \\
\hline Lack of efficacy & - & - & - & - & 1 & 0.7 & 1 & 0.2 \\
\hline Reason for treatment withdrawal & 61 & 40.4 & 67 & 44.4 & 57 & 38.0 & 185 & 40.9 \\
\hline Non medical reason & 39 & 25.8 & 39 & 25.8 & 35 & 23.3 & 113 & 25.0 \\
\hline Adverse event & 18 & 11.9 & 25 & 16.6 & 18 & 12.0 & 61 & 13.5 \\
\hline Protocol deviation & 2 & 1.3 & 2 & 1.3 & 1 & 0.7 & 5 & 1.1 \\
\hline Lost to follow up & 2 & 1.3 & 1 & 0.7 & 2 & 1.3 & 5 & 1.1 \\
\hline Lack of efficacy & - & - & - & - & 1 & 0.7 & 1 & 0.2 \\
\hline Completed & 92 & 60.9 & 87 & 57.6 & 100 & 66.7 & 279 & 61.7 \\
\hline In compliance with the protocol & 64 & 42.4 & 52 & 34.4 & 61 & 40.7 & 177 & 39.2 \\
\hline $\begin{array}{l}\text { With a protocol deviation after } \\
\text { inclusion }\end{array}$ & 28 & 18.5 & 35 & 23.2 & 39 & 26.0 & 102 & 22.6 \\
\hline Analysis Set & & & & & & & & \\
\hline Randomised Set (RS) & 151 & 100 & 151 & 100 & 150 & 100 & 452 & 100 \\
\hline Safety Set (SS) & 151 & 100 & 149 & 98.7 & 150 & 100 & 450 & 99.6 \\
\hline Full Analysis Set (FAS) & 119 & 78.8 & 112 & 74.2 & 126 & 84.0 & 357 & 79.0 \\
\hline
\end{tabular}

A total of 452 patients were included and randomly assigned to one of the 3 treatment groups, with a well balanced distribution: 151 patients in the SrRan 1g group, 151 in the SrRan 2g group, and 150 in the placebo group. Of them, 173 patients (38.3\% of the randomised patients) withdrew the study, with a higher frequency reported in the SrRan treatment groups than in the placebo group (39.1\% in the SrRan $1 \mathrm{~g}$ group, $42.4 \%$ in the SrRan $2 \mathrm{~g}$ group, and $33.3 \%$, in the placebo group), mainly due to non-medical reasons (111 patients, $24.6 \%)$, with a higher frequency reported in the SrRan groups than in the placebo group $(25.2 \%, 26.5 \%$, versus $22.0 \%$, respectively). At inclusion, 61 patients (13.5\%) reported at least one protocol deviation, that concerned mainly study management (6.6\%). During the study, 205 patients $(45.4 \%)$ reported at least one protocol deviation, that concerned mainly study management (26.3\%). No relevant between-group difference was observed for protocol deviations.

\section{BASELINE CHARACTERISTICS}

Patients were in average $60.5 \pm 7.0$ years old and most of them (46.2\%) were aged between 55 and 65 years. A majority was women $(85.4 \%)$. BMI was in average $25.3 \pm 3.5 \mathrm{~kg} / \mathrm{m}^{2}$. No relevant between-group differences were observed.

Patients were suffering from knee OA for $35.7 \pm 46.5$ months in average (about 3 years) (median $=18$ months). Kellgren and Lawrence score on the target knee was assessed at stage II for a majority of patients $(74.6 \%)$, and stage III for $25.4 \%$ of them, with a higher frequency of patients affected with stage III in the SrRan $1 \mathrm{~g}$ group than in the SrRan $2 \mathrm{~g}$ and placebo groups $(29.1 \%, 24.5 \%$, and $22.7 \%$, respectively). At the physical assessment, patients had swelling $(19.9 \%$, with a higher frequency reported in the SrRan $1 \mathrm{~g}$ group than in the SrRan $2 \mathrm{~g}$ and placebo groups: $24.5 \%, 16.6 \%, 18.7 \%)$, effusion $(11.3 \%$, with a higher frequency reported in the SrRan $1 \mathrm{~g}$ group than in the SrRan $2 \mathrm{~g}$ and placebo groups: $13.9 \%, 8.6 \%, 11.3 \%$, respectively), warmth (5.8\%), or other symptoms $(98.4 \%)$. 


\section{SUMMARY - CONCLUSIONS (Cont'd)}

A total of $27.2 \%$ of the patients were receiving a treatment for $\mathrm{OA}$ at inclusion, with a higher frequency in the SrRan groups than in the other groups, particularly marked in the SrRan 1g group: $31.1 \%$ in the SrRan $1 \mathrm{~g}$ group, $27.2 \%$ in the SrRan $2 \mathrm{~g}$ group, and $23.3 \%$ in the placebo group. These treatments were mainly Antiinflammatory and antirheumatic products (24.3\%), and Analgesics (5.3\%). Most of the patients (95.1\%) reported a medical history other than the knee OA, mainly menopause (69.7\%) and hypertension (33.2\%).

Efficacy parameters at baseline were comparable across treatment groups: the knee mean value of JSW was $3.52 \pm 0.77 \mathrm{~mm}$, with a mean VAS pain of the target knee of 55.2 $\pm 18.2 \mathrm{~mm}$ (on a 0-100 scale score), and the mean knee WOMAC global score was $113.8 \pm 57.7$ (on a 0-300 scale score).

\section{EXTENT OF EXPOSURE}

In the Safety Set, the mean treatment duration was $874.7 \pm 375.0$ days, i.e. approximately 2.4 years. The exposure to the treatment was close to the treatment duration: $857.4 \pm 374.2$ days (i.e. 2.3 years), indicating that only few patients interrupted the treatment and for short periods of time. The global compliance was high: $94.8 \pm 10.9 \%$, in the Safety Set. Most of the patients $(74.2 \%)$ were treated for at least 2 years. No relevant between-group difference was observed.

\section{EFFICACY RESULTS}

Primary assessment criterion: minimum JSW of the knee

Knee JSW (mm) reading by PMO - Change from baseline to End - FAS (N = 357)

\begin{tabular}{|c|c|c|c|c|}
\hline Knee JSW (mm) & & $\begin{array}{l}\text { SrRan 1g } \\
(\mathrm{N}=119)\end{array}$ & $\begin{array}{l}\text { SrRan 2g } \\
(\mathrm{N}=112)\end{array}$ & $\begin{array}{c}\text { Placebo } \\
(\mathrm{N}=126)\end{array}$ \\
\hline & $\mathrm{n}$ & 119 & 112 & 126 \\
\hline \multirow[t]{3}{*}{ Baseline } & Mean \pm SD & $3.5036 \pm 0.7520$ & $3.5032 \pm 0.7304$ & $3.5182 \pm 0.8347$ \\
\hline & Median & 3.5790 & 3.4820 & 3.4710 \\
\hline & Min ; Max & $1.409 ; 5.064$ & $1.898 ; 5.189$ & $0.632 ; 5.856$ \\
\hline \multirow[t]{3}{*}{ END } & Mean \pm SD & $3.2550 \pm 1.1127$ & $3.3463 \pm 0.9568$ & $3.3592 \pm 0.9835$ \\
\hline & Median & 3.4170 & 3.4170 & 3.4355 \\
\hline & Min ; Max & $0.636 ; 6.622$ & $1.125 ; 5.658$ & $0.624 ; 5.844$ \\
\hline \multirow[t]{3}{*}{ Change from baseline to End } & Mean \pm SD & $-0.2486 \pm 0.7253$ & $-0.1568 \pm 0.7045$ & $-0.1590 \pm 0.5855$ \\
\hline & Median & -0.2120 & -0.0385 & -0.0545 \\
\hline & Min ; Max & $-3.203 ; 2.392$ & $-3.077 ; 1.206$ & $-3.181 ; 1.712$ \\
\hline \multirow{3}{*}{ Statistical analysis } & $\mathrm{E}(\mathrm{SE})^{(1)}$ & $-0.089(0.086)$ & $-0.015(0.088)$ & - \\
\hline & $95 \% \mathrm{CI}^{(2)}$ & {$[-0.282 ; 0.103]$} & {$[-0.210 ; 0.181]$} & \\
\hline & p-value ${ }^{(3)}$ & 0.483 & 0.980 & \\
\hline
\end{tabular}

$N$ : total number of patients in each treatment group considered; $n:$ number of assessable patients; NA: Not Applicable; End: last reliable post-baseline value; Estimate (Standard error) of the difference between baseline gender and centre adjusted treatment group means : SrRan $1 \mathrm{~g}$ or $2 \mathrm{~g}$ minus placebo; (1) Dunnetts $95 \%$ Confidence interval of the estimate; (2) Dunnetts adjusted p-value (General linear model with baseline as covariate and gender and centre as fixed factor)

A decrease in the knee JSW was observed from baseline to end in all treatment groups $(-0.25 \pm 0.73 \mathrm{~mm}$ in the SrRan $1 \mathrm{~g}$ group, $-0.16 \pm 0.70 \mathrm{~mm}$ in the SrRan $2 \mathrm{~g}$ group, and $-0.16 \pm 0.59 \mathrm{~mm}$ in the placebo group), and no statistically significant between-group difference was detected between the SrRan $1 \mathrm{~g}$ or $2 \mathrm{~g}$ group and the placebo group.

\section{Secondary criteria}

- Radiological and radio-clinical progression of the knee osteoarthritis

Radiological failures (defined as a JSW loss $\geq 0.5 \mathrm{~mm}$ ) were reported in $26.1 \%$ in the SrRan $1 \mathrm{~g}$ group, $21.4 \%$ in the SrRan $2 \mathrm{~g}$ group and $23.8 \%$ in the placebo group, and radio-clinical failures (defined as a JSW loss $\geq 0.5 \mathrm{~mm}$ and a WOMAC sub-score pain improvement $\leq 20 \%$ ) in $11.4 \%, 6.3 \%$ and $8.0 \%$, respectively.

\section{- Clinical assessments}

\section{WOMAC global score for the target knee}

The WOMAC global score decreased in all treatment groups as well as in each component, showing an improvement of the patients. A tendency to a larger improvement was observed in the SrRan $2 \mathrm{~g}$ group compared to the placebo group (mean change from baseline to End: $-40.83 \pm 69.85 \mathrm{~mm}$ in the SrRan $1 \mathrm{~g}$ group, $-53.21 \pm 64.69 \mathrm{~mm}$ in the SrRan $2 \mathrm{~g}$ group, and $-42.65 \pm 70.28 \mathrm{~mm}$ in the placebo group). 


\section{SUMMARY - CONCLUSIONS (Cont'd)}

The WOMAC sub-scores showed the same trends (WOMAC pain sub-score: $-14.61 \pm 23.93 \mathrm{~mm}$, $-20.52 \pm 22.06 \mathrm{mmg}$, and $-16.05 \pm 26.29 \mathrm{~mm}$, respectively, WOMAC stiffness sub-score: $-12.85 \pm 26.74 \mathrm{~mm}$, $-16.18 \pm 24.86$, and $-12.61 \pm 22.09 \mathrm{~mm}$, respectively, WOMAC physical function sub-score: $-12.52 \pm 25.51 \mathrm{~mm},-16.57 \pm 23.03 \mathrm{~mm}$, and $-13.99 \pm 27.26 \mathrm{~mm}$, respectively). The rate of responders for the WOMAC pain sub-score (defined as an improvement of $20 \%$ ) was high in all treatment groups, reflecting a large placebo effect $(88.1 \%, 90.2 \%$ and $84.8 \%$, respectively).

\section{- Visual Analogic Score for the target knee pain}

The mean VAS decreased in all treatment groups, showing, as for the WOMAC, a placebo effect. The improvement tended to be slightly larger in the SrRan treatment groups than in the placebo group $(-30.9 \pm 27.7 \mathrm{~mm}$ in the SrRan $1 \mathrm{~g}$ group, $-31.5 \pm 24.9 \mathrm{~mm}$ in the SrRan $2 \mathrm{~g}$ group, and $-27.0 \pm 25.4 \mathrm{~mm}$ in the placebo group).

Other secondary criteria did not show any relevant between-group differences.

\section{SAFETY RESULTS}

\section{Adverse events}

\begin{tabular}{lcccc}
\multicolumn{5}{c}{ Summary of safety results - Safety Set $\mathbf{( N = 4 5 0 )}$} \\
\hline
\end{tabular}

Emergent adverse events (EAEs) were reported with no clear relevant between-groups difference in the frequencies $(67.5 \%, 73.2 \%$ and $70.0 \%$, respectively). The most frequently affected system organ classes in all treatment groups were Gastrointestinal disorders, with a higher frequency of patients affected reported in the SrRan treatment groups than in the placebo group (29.8\%, 31.5\% and $22.7 \%$, respectively), Infections and infestations $(25.2 \%, 28.2 \%$, and $29.3 \%$, respectively), and Musculoskeletal and connective tissue disorders, with a higher frequency of patients affected reported in the SrRan treatment groups than in the placebo group $(30.5 \%, 27.5 \%$, and $28.0 \%$, respectively).

The most frequently reported EAEs (at least $10 \%$ in either treatment group) were the following: osteoarthritis, with a higher frequency reported in the SrRan $2 \mathrm{~g}$ group than in the placebo group (9.9\% in the $\mathrm{SrRan} 1 \mathrm{~g}$ group, $14.1 \%$ in the SrRan $2 \mathrm{~g}$ and $10.7 \%$ in the placebo group), upper respiratory tract infection $(7.9 \%$, $12.8 \%$, and $12.0 \%$, respectively), and nasopharyngitis $(9.3 \%, 10.7 \%$ and $12.0 \%$, respectively). The EAEs more frequently reported in the SrRan $1 \mathrm{~g}$ and/or $2 \mathrm{~g}$ groups than in the placebo group (difference of more than $2 \%$ between one of the SrRan treatment group and the placebo group, ordered by decreasing order of the between-group difference SrRan $1 \mathrm{~g}$ or $2 \mathrm{~g}$ group versus placebo group) were mainly the following: blood creatine phosphokinase increased $(4.0 \%, 5.4 \%$, versus $1.3 \%$, respectively), hypertension $(6.6 \%, 8.7 \%$ versus $4.7 \%$, respectively), osteoarthritis $(9.9 \%, 14.1 \%$, versus $10.7 \%$, respectively), and dizziness $(3.3 \%$, $5.4 \%$ versus $2.0 \%$, respectively).

As regards known important events listed in the current SmPC of the strontium ranelate, no case of emergent Drug Rash with Eosinophilia and Systemic Symptoms (DRESS) or Stevens-Johnson syndrome, nor toxic epidermal necrolysis, nor venous thromboembolic events (deep vein thrombosis, pulmonary embolism), nor myocardial infarction was reported.

EAEs were severe in $3.1 \%, 4.3 \%$, and $5.8 \%$ of the EAEs, respectively, mainly the SOC Infections and infestations in the 3 treatment groups (2.0\% in each SrRan group and $2.7 \%$ in the placebo group).

Treatment-related EAEs were reported with a higher frequency in the SrRan $2 \mathrm{~g}$ group than in the placebo group: $19.2 \%$ in the SrRan $1 \mathrm{~g}$ group, $28.2 \%$ in the SrRan $2 \mathrm{~g}$ group and $23.3 \%$ in the placebo group. The SOCs most commonly affected in the three groups were Gastrointestinal disorders $(11.3 \%, 10.1 \%$ and $10.7 \%$ in the placebo group), Investigations with a higher frequency reported in the SrRan $1 \mathrm{~g}$ and $2 \mathrm{~g}$ groups than in the placebo group $(4.6 \%, 7.4 \%$, and $2.7 \%$, respectively), and Skin and subcutaneous tissue disorders $(3.3 \%$, $7.4 \%$ and $6.0 \%$, respectively). 


\section{SUMMARY - CONCLUSIONS (Cont'd)}

The frequency of patients who reported at least one EAE leading to treatment withdrawal was higher in the SrRan 2g group than in the other groups: $11.9 \%$ in the SrRan $1 \mathrm{~g}$ group, $15.4 \%$ in the SrRan $2 \mathrm{~g}$ group, and $11.3 \%$ in the placebo group. The most frequently affected SOC was Gastrointestinal disorders: $4.6 \%, 4.7 \%$ and $1.3 \%$, respectively. The EAEs considered as treatment-related or leading to treatment withdrawal were principally those usually known with strontium ranelate.

Emergent serious AEs were reported with a higher frequency in the SrRan $2 \mathrm{~g}$ group than in the other tretament groups: $8.6 \%$ in the SrRan $1 \mathrm{~g}$ group, $10.7 \%$ in the SrRan $2 \mathrm{~g}$ group versus $8.0 \%$ in the placebo group. The most frequently affected SOCs in the SrRan $1 \mathrm{~g}$ group were Musculoskeletal and connective tissue disorders $(2.6 \%, 1.3 \%$, and $2.0 \%)$, and Infections and infestations $(2.6 \%, 1.3 \%$, and $1.3 \%$, respectively). The most frequently affected SOC in the SrRan $2 \mathrm{~g}$ group was Injury, poisoning and procedural complications: $0.7 \%$ in the SrRan $1 \mathrm{~g}$ group, $2.0 \%$ in the SrRan $2 \mathrm{~g}$ group, and $1.3 \%$ in the placebo group. No particular event (preferred term) was affected by the emergent serious adverse events.

A total of 3 serious EAEs in 3 patients were considered as treatment-related by the investigator (bronchitis in one patient in the SrRan $1 \mathrm{~g}$ group, and supraventricular tachycardia and cerebral ischaemia in one patient each in the placebo group). These serious EAEs were recovered (bronchitis, supraventricular tachycardia) or recovering/improving (cerebral ischaemia).

During the study, 2 patients died: 1 in the SrRan $2 \mathrm{~g}$ group (death, for which no information was available), and 1 in the placebo group (sudden death, considered by the investigator as related to medical history).

\section{Laboratory tests}

No relevant change over time nor between-group difference was detected for biochemistry or heamatology parameters, except for CPK, with a mean increase from baseline to end larger in the SrRan groups than in the placebo group: $11.8 \pm 47.6 \mathrm{IU} / \mathrm{L}$ in the SrRan $1 \mathrm{~g}$ group, $12.4 \pm 53.4 \mathrm{IU} / \mathrm{L}$ in the SrRan $2 \mathrm{~g}$ group, and $7.3 \pm 41.0 \mathrm{IU} / \mathrm{L}$ in the placebo group.

Emergent potentially clinically significant abnormal values (PCSAs) were sparse ( $<5 \%$ in any group) for biochemistry parameters except for phosphorus, for which high PCSA values were more frequently reported in the SrRan groups than in the placebo group $(17.4 \%, 26.1 \%$ versus $7.6 \%$, respectively), and $\mathrm{C}$ reactive protein $(12.1 \%, 9.7 \%$, versus $13.9 \%$, respectively).

Emergent PCSAs were sparse for haematology parameters except for prothrombin time (low values: 19.5\%, $16.4 \%$, and $17.4 \%$, respectively), and activated partial thromboplastin time (high values: $41.5 \%, 37.9 \%$, and $38.8 \%$, respectively).

Vital signs

Neither clinically relevant changes over time nor differences between groups were detected.

\section{CONCLUSION}

This international double-blind controlled phase III study, was conducted in 452 Asian patients with primary knee osteoarthritis, over a 3-year treatment period. A decrease in the knee JSW (primary efficacy criterion) was observed from baseline to end in the 3 treatment groups, and no statistically significant difference between SrRan groups versus placebo was detected. The analysis of the radiological and radio-clinical failures did not show a clinically relevant SrRan treatment versus placebo effect. An improvement in symptoms of osteoarthritis, was detected in the three treatment groups, as evaluated on the target knee by the WOMAC global score and sub-scores. A larger effect was observed in the SrRan $2 \mathrm{~g}$ group than in the other groups. A trend in favour of SrRan $1 \mathrm{~g}$ and $2 \mathrm{~g}$ was found for the algo-functional assessment of the target knee (VAS pain scale). The overall incidence of emergent adverse events did not show a clear relevant between-group difference. The incidence of serious emergent adverse events was larger in the SrRan $2 \mathrm{~g}$ group than in the other treatment groups, but no particular safety concern was observed. With regards to known important events listed in the current SmPC of the strontium ranelate, no case of emergent Drug Rash with Eosinophilia and Systemic Symptoms (DRESS) or Stevens-Johnson syndrome, nor toxic epidermal necrolysis, nor venous thromboembolic events (deep vein thrombosis, pulmonary embolism), nor myocardial infarction was reported. The safety profile of SrRan $2 \mathrm{~g} / \mathrm{day}$ in patients with OA was similar with that previously described in osteoporotic patients.

Date of the report: 25 November 2014

Version of the report: Final version 